\title{
Postoperative stereotactic radiosurgery for resected brain metastases: A comparison of outcomes for large resection cavities
}

\author{
Jim Zhong, MDa, ${ }^{\mathrm{*}}$, Matthew J. Ferris, MD $^{\mathrm{a}}$, Jeffrey Switchenko, PhD $^{\mathrm{b}}$, Robert H. Press, MD ${ }^{\mathrm{a}}$, \\ Zachary Buchwald, MD, PhDa, Jeffrey J. Olson, MD $^{\mathrm{c}}$, Bree R. Eaton, MD ${ }^{\mathrm{a}}$, Walter J. Curran, \\ MDa, Hui-Kuo G. Shu, MD, PhDa, lan R. Crocker, MDa ${ }^{a}$, and Kirtesh R. Patel, MD ${ }^{a, d}$ \\ aDepartment of Radiation Oncology, Winship Cancer Institute, Emory University, Atlanta, Georgia \\ bBiostatistics and Bioinformatics Shared Resource, Winship Cancer Institute, Emory University, \\ Atlanta, Georgia \\ 'Department of Neurosurgery, Winship Cancer Institute, Emory University, Atlanta, Georgia \\ ${ }^{\mathrm{d}}$ Department of Radiation Oncology, Veterans Affairs Hospital, Decatur, Georgia
}

\begin{abstract}
Purpose-Although historical trials have established the role of surgical resection followed by whole brain irradiation (WBRT) for brain metastases, WBRT has recently been shown to cause significant neurocognitive decline. Many practitioners have employed postoperative stereotactic radiosurgery (SRS) to tumor resection cavities to increase local control without causing significant neurocognitive sequelae. However, studies analyzing outcomes of large brain metastases treated with resection and postoperative SRS are lacking. Here we compare outcomes in patients with large brain metastases $>4 \mathrm{~cm}$ to those with smaller metastases $\leqslant 4 \mathrm{~cm}$ treated with surgical resection followed by SRS to the resection cavity.
\end{abstract}

Methods and materials-Consecutive patients with brain metastases treated at our institution with surgical resection and postoperative SRS were retrospectively reviewed. Patients were stratified into $\$ 4 \mathrm{~cm}$ and $>4 \mathrm{~cm}$ cohorts based on preoperative maximal tumor dimension. Cumulative incidence of local failure, radiation necrosis, and death were analyzed for the 2 cohorts using a competing-risk model, defined as the time from SRS treatment date to the measured event, death, or last follow-up.

Results-A total of 117 consecutive cases were identified. Of these patients, 90 (77\%) had preoperative tumors $\leq 4 \mathrm{~cm}$, and $27(23 \%)>4 \mathrm{~cm}$ in greatest dimension. The only significant baseline difference between the 2 groups was a higher proportion of patients who underwent gross total resection in the $\$ 4 \mathrm{~cm}$ compared with the $>4 \mathrm{~cm}$ cohort, $76 \%$ versus $48 \%$, respectively $(P<$. 01 ). The 1-year rates of local failure, radiation necrosis, and overall survival for the $\leq 4 \mathrm{~cm}$ and $>4$ cm cohorts were $12.3 \%$ and $16.0 \%, 26.9 \%$ and $28.4 \%$, and $80.6 \%$ and $67.6 \%$, respectively (all $P$

*Corresponding author. Department of Radiation Oncology, Emory University, 1365 Clifton Rd NE, Room AT225, Atlanta, GA 30322.Jim.Zhong@emory.edu (J. Zhong).

Conflicts of interest: None. 
>.05). The rates of local failure and radiation necrosis were not statistically different on multivariable analysis based on tumor size.

Conclusions-Brain metastases $>4 \mathrm{~cm}$ in largest dimension managed by resection and radiosurgery to the tumor cavity have promising local control rates without a significant increase in radiation necrosis on our retrospective review.

\section{Introduction}

Metastatic disease continues to represent the most common etiology for brain tumors, with more than 170,000 cases diagnosed per year. ${ }^{1}$ The incidence of brain metastases has also been rising, in part because of improvements in systemic therapy for extracranial disease. ${ }^{2}$ For patients with either symptomatic or large brain metastases, surgical resection has generally been the preferred initial treatment. Historical trials have established the role of postoperative whole brain irradiation (WBRT) to improve local and distant brain control. ${ }^{3-5}$ Postoperative WBRT, however, does not improve overall survival (OS), ${ }^{6}$ and with recent evidence demonstrating neurocognitive decline following WBRT, its use has been decreasing. ${ }^{7-9}$

Several prospective phase 3 trials have evaluated the efficacy of stereotactic radiosurgery (SRS) to intact brain metastases, demonstrating excellent local control (LC). ${ }^{3,10,11}$ This paradigm of focal irradiation has been subsequently applied to the postoperative resection cavity ${ }^{12-14}$ to improve LC in efforts to avoid the neurocognitive sequelae seen with WBRT. ${ }^{15}$ As such, many clinicians have extrapolated postoperative treatment techniques from data generated by intact-lesion SRS studies, which generally excluded enrollment of patients with metastases $>3$ to $4 \mathrm{~cm}$. $3,10,11$

Consequently, for those patients with brain metastases $>4 \mathrm{~cm}$, few data are available that predicts their outcome when treated with surgical resection and adjuvant radiosurgery. Without this information, practitioners may resort to WBRT even for patients with high performance statuses, fearing inadequate LC or excess toxicity when treating large resection cavities. Here, we retrospectively compare outcomes in patients with large brain metastases $>4 \mathrm{~cm}$ with those with smaller tumors $\leqslant 4 \mathrm{~cm}$ treated with surgical resection followed by SRS to the resection cavity.

\section{Methods and materials}

\section{Patients}

All patients treated in the Emory Healthcare system from 2006 through 2015 with a diagnosis of brain metastasis were analyzed with the Emory institutional review board approval. Only those patients treated with surgical resection of their metastatic intracranial disease followed by adjuvant SRS alone to the resection cavity were included for analysis. Patients treated with prior WBRT were not eligible. Radiosensitive histologies (ie, small-cell lung cancer, lymphoma, and germ cell) were excluded.

Patient and tumor characteristics including patient's age at diagnosis, gender, primary tumor histology, preoperative tumor dimension, number of brain metastases, date of surgical 
resection, the extent of resection, date of radiosurgery, and radiosurgery parameters including radiation dose, the number of fractions, and planning tumor volume (PTV) margin were retrospectively reviewed. Patient's recursive partitioning analysis and graded prognostic assessment scores were calculated retrospectively based on chart review.

\section{Treatment and follow-up}

The decision for surgical resection was made by the treating neurosurgeon on the basis of tumor size, location, associated symptoms, and need for pathologic diagnosis. Neurosurgical resection was then performed by using standard technique; gross total resection of each lesion was achieved whenever possible.

Patients received postoperative SRS approximately 2 to 4 weeks following surgical resection. Patients were treated with LINAC-based radiosurgery, either framed or frameless. Patients were simulated using a computed tomography scan with either a thermoplastic head mask (for frameless cases), or a stereotactic headframe (for framed cases) placed by a neurosurgeon. The computed tomography simulation images were subsequently registered to the patient's brain magnetic resonance imaging (MRI), obtained within 14 days of radiation planning, using the Velocity registration software. Gross tumor volume (GTV) was delineated using MRI. The GTV was uniformly expanded to directly from the GTV without a clinical target volume margin. The PTV margin varied based on physician preference and whether stereotactic headframe was used. Six-megavolt photons were used. Treatment planning for frameless and framed radiosurgery used the Eclipse and Brainlab planning softwares, respectively.

Follow-up after treatment consisted of clinical examination and MRI of the brain with and without contrast 4 to 6 weeks after postoperative SRS; subsequent follow-up consisted clinical examination and MRI brain imaging every 3 to 4 months thereafter, unless clinically indicated at an earlier time point. Toxicity was retrospectively graded using the Common Terminology Criteria for Adverse Events, version 4.0.

\section{Statistical analysis}

Local recurrence (LR) was the primary outcome, defined as recurrence seen within the prior $80 \%$ isodose line on follow-up MRI at or near the resection cavity. LR was calculated using a competing-risk model, defined as the time from SRS treatment date to LR, death, or last imaging follow-up; death without LR was considered a competing event. The time to development of radiation necrosis was also calculated using the competing-risk model. All intracranial outcomes analyses were performed at the cavity/lesion level. Fine and Gray's method was used for multivariable analysis of competing risk endpoints.

Radiation necrosis was defined on the basis of 2 radiographic features: the development of contrast enhancing mass within prior radiation treatment fields and conventional imaging features, including soap bubble appearance. ${ }^{16}$ If there was a question of the enhancement representing LR or radiation necrosis, additional advanced imaging (eg, magnetic resonance perfusion, magnetic resonance spectroscopy, brain positron emission tomography) was obtained and consensus was reached in a multidisciplinary neuro-oncology tumor board. In cases where additional imaging was still equivocal, surgical resection was performed. 
For OS, death from any cause was defined as the event, and patients were censored at time of last known alive. OS was estimated by the Kaplan-Meier product-limit method; the logrank test was used to assess for differences between treatment groups. ${ }^{17}$ Univariable and multivariable analysis (MVA) was performed using the Cox proportional hazards model.

Univariable and multivariable analysis for covariates were performed for LR, radiation necrosis, and OS. The variables found to have a $P$ value of .1 or less on univariate analysis were then used in MVA. For those outcomes for which there were fewer than four variables with $P$ values more significant than .1, the 4 variables with the most significant $P$ values on univariate analysis were used in multivariable analysis. All statistical analyses were 2-sided, with $P<.05$ considered statistically significant. Statistical analysis was performed using the SAS 9.4 statistical software package.

\section{Results}

\section{Baseline patient and treatment characteristics}

From 2006 through 2015, 117 consecutive cases of resected brain metastases treated with postoperative radiosurgery were identified at our institution. Of these patients, $90(77 \%)$ had preoperative tumors $\leq 4 \mathrm{~cm}$ and $27(23 \%)$ had preoperative tumors $>4 \mathrm{~cm}$ in greatest dimension. Baseline patient and tumor characteristics between these 2 groups are displayed in Table 1 . The median follow-up time was 24 months. The only significant baseline difference between these groups was a higher proportion of patients with gross total resection of their tumors in the $\leq 4 \mathrm{~cm}$ compared with the $>4 \mathrm{~cm}$ cohort, 68 (76\%) versus 13 (48\%), respectively $(P<.01)$. Also, as expected, the prescribed PTV volume in the $>4 \mathrm{~cm}$ cohort was significantly larger than the $\leqslant 4 \mathrm{~cm}$ cohort, mean $33.7 \mathrm{~mL}$ (95\% confidence interval [CI], 26.6-40.1) versus $18.6 \mathrm{~mL}$ (95\% CI, 16.0-21.2) $(P<.001)$, respectively. The mean GTV in the $>4 \mathrm{~cm}$ cohort was also significantly larger as expected compared with the $\lessgtr 4 \mathrm{~cm}$ cohort: $20.8 \mathrm{~mL}$ vs $11.1 \mathrm{~mL}(P<.001)$. No significant differences in treatment characteristics, including the prevalence of framed versus frameless radiosurgery, total prescribed dose, PTV margin, or the number of prescribed dose in the 2 cohorts were identified (all $P>.05$ ). The mean prescribed biologically effective dose at alpha/beta ratio of 10 (was significantly lower for the $>4 \mathrm{~cm}$ cohort, $42.5 \mathrm{~Gy}$ vs $46.8 \mathrm{~Gy}, P=.007$. The mean BED used at each fractionation regimen is summarized in Table 1.

\section{LC}

Table 2 demonstrates the univariate association of patient and tumor characteristics with LR. The 1-year LR rates were $12.3 \%$ (95\% CI, 6.2-20.5) and $16.0 \%$ (95\% CI, 4.85-33.0) for the $\checkmark 4 \mathrm{~cm}>4 \mathrm{~cm}$ cohorts, respectively $(P=.60)$. The median time to LR has not been reached for either cohort. On univariate analysis, no significant association between the tumor size cohorts and LR was found (hazard ratio [HR], 1.33 [95\% CI, 0.47-3.73]; $P=.59$ ). Only older age at diagnosis was significantly associated with lower LR (HR, 0.96; 95\% CI, 0.96 [0.91-1.00]; $P=.04)$. The primary site of disease, graded prognostic assessment score, the number of treatment fractions, extent of resection, prescription dose and the use of a stereotactic headframe were not significantly associated with LC (all $P>.05$ ). Tumor size, patient age, primary tumor site, and dose per fraction were used in the multivariable 
calculation; no variable was found to be significantly associated with LR on MVA (all $P>$. 05). Cumulative incidence curves for the development of LR are depicted in Fig 1.

Distant intracranial failure rates for the $\leq 4 \mathrm{~cm}$ and $>4 \mathrm{~cm}$ cohorts were $46.7 \%$ (95\% CI, 35.9-56.9) versus 55.6\% (95\% CI, 34.6-72.2) at 1 year and 56.6\% (95\% CI, 45.3-66.4) versus $64.5 \%(95 \% \mathrm{CI}, 41.6-80.3)$ at 2 years, respectively $(P=.08)$.

\section{Radiation necrosis}

The univariate associations of patient and tumor characteristics with development of radiation necrosis are listed in Table 2 . The 1-year cumulative incidence rates of radiation necrosis were $26.9 \%(17.7-36.9)$ and $28.4 \%$ (95\% CI, 12.2-47.2), for the $\$ 4 \mathrm{~cm}$ and $>4 \mathrm{~cm}$ cohorts, respectively $(P=.92)$. The percentage of radiation necrosis that was symptomatic were $67 \%$ (26 of 39) and 54\% (6 of 11) in the $\leq 4 \mathrm{~cm}$ and $>4 \mathrm{~cm}$ cohorts, respectively ( $P=$. 46 ), corresponding to 1 -year rates of symptomatic radiation necrosis of $20.5 \%$ and $20.1 \%$, respectively $(P=.95)$. Of the 32 patients who received treatment for radiation necrosis, all were managed with corticosteroids or hyperbaric oxygen with no patient demonstrating Common Terminology Criteria for Adverse Events grade 3 or higher toxicity. On univariate analysis, preoperative tumor size was not significantly associated with the development of radiation necrosis (HR, 0.99 [95\% CI, 0.50-1.98]; $P=.99$ ). Additionally, PTV size, prescription dose, dose per fraction, and PTV margin were also not significantly associated with the development of radiation necrosis (all $P>.05$ ). Three factors were associated with radiation necrosis: non-small cell lung primary histology (HR, 2.65 [95\% CI, 10.2-6.88]; $P$ $=.44)$, older age (HR, 1.03 [95\% CI, 1.01-1.06]; $P=.017)$, and frameless radiosurgery (HR, 0.52 [95\% CI, 0.27-1.00]; $P=.05$ ). Tumor size, patient age, primary tumor site, and receipt of frameless radiosurgery were used in MVA; frameless radiosurgery was significantly associated with decreased rates of radiation necrosis (HR, 0.49 [95\% CI, 0.25-0.97]; $P=$. 042). Preoperative tumor size $>4 \mathrm{~cm}$ was not associated with radiation necrosis on MVA.

OS

There were no significant differences in OS among the $\leqslant 4 \mathrm{~cm}$ and $>4 \mathrm{~cm}$ cohorts, with 1year OS of $80.6 \%$ (95\% CI, 70.2-87.6) and 67.6\% (95\% CI, 45.4-82.3), respectively ( $P=$. 21). The univariate associations of patient and disease characteristics with death are demonstrated in Table 2. Patient age trended toward significance in its association with death on univariable analysis (HR, 0.98 [95\% CI, 0.96-1.00]; $P=.06$ ) and was found to be significant on MVA (HR, 0.98 [95\% CI, 0.96-1.00; $P=.048$ ). No other factors were found to be significantly associated with OS on multivariable analysis.

\section{Leptomeningeal disease}

The cumulative rates of leptomeningeal disease in the $₫ 4 \mathrm{~cm}$ and $>4 \mathrm{~cm}$ cohorts were $13.1 \%$ (95\% CI, 6.9-21.3) versus $15.1 \%$ (95\% CI, 4.6-31.4) at 1 year and $22.8 \%$ (95\% CI, 12.132.7 ) versus $19.8 \%$ (95\% CI, 6.8-37.7), respectively $(P=.72)$. On multivariate analysis, neither preoperative lesion size nor PTV was not significantly associated with the development of leptomeningeal disease $(P>.05)$. 


\section{Discussion}

A main challenge of radiation therapy in treatment of brain metastases is the achievement of durable LC while limiting normal tissue toxicity. ${ }^{18}$ The purpose of this study was to evaluate the efficacy of radiosurgery for resection cavities of large tumors. Our results demonstrate promising LC rates in resection cavities treated with postoperative SRS regardless of initial tumor size. Additionally, increased treatment volume size was not significantly associated with rates of radiation necrosis in our series.

Most recently, Mahajan et al reported the results of a single institutional prospective randomized trial evaluating the LC rates of resection of brain metastases following surgical resection followed by SRS versus observation at the 2016 American Society of Therapeutic Radiation Oncology annual meeting. ${ }^{19}$ All patients on this study received single-fraction SRS, with doses based on volume (ie, <10mL, 16 Gy; $10.115 \mathrm{~mL}, 14 \mathrm{~Gy}$; >15 mL, 12 Gy). When stratified by tumor size, their reported 1-year LC rates were significantly lower for those tumors $>3.5 \mathrm{~cm}$ compared with $2.5 \mathrm{~cm}$ (46\% vs $91 \%, P=.004)$. Similarly, Ballman et al also recently reported in abstract form the results of the N107C.CEC.3 study, comparing adjuvant SRS to WBRT in resected brain metastases. ${ }^{15}$ These authors also report relatively poor 1-year LC rates of $55.6 \%$ in their entire SRS cohort, which was not stratified by size.

Our results appear different from these findings, as we found no difference in LC for our 2 size cohorts, and our 1-year LC rates was $84 \%$ for our $>4 \mathrm{~cm}$ cohort. Our treatment approach differed in a few ways from these 2 studies. The mean PTV for our $>4 \mathrm{~cm}$ cohort was $33.7 \mathrm{~mL}$, and when these cavities were treated with single fraction SRS, doses of 15 to 18 Gy were prescribed. These prescription doses are higher than those of the MD Anderson and N107C.CEC. 3 trial specifications of $12 \mathrm{~Gy}$ for $>$ PTV $15 \mathrm{~mL}$ and PTV $230 \mathrm{~mL}$, respectively. ${ }^{20}$ It may be that our relative dose escalation may allow for increased LC. Additionally, in our series, $48 \%$ of patients in the $>4 \mathrm{~cm}$ cohort were treated with hypofractionated SRS, ${ }^{21}$ and Minitti et al report that for resection cavities $>2.0 \mathrm{~cm}$, LC may be higher with hypofractionated radiation compared with single-fraction SRS. ${ }^{22}$

PTV margins may also be an important factor for LC. The Stanford group demonstrated that $2 \mathrm{~mm}$ margin added to resection cavities resulted in improved LC compared with $0 \mathrm{~mm}$ margin. ${ }^{23}$ Since the publication of this study, our practice has changed from $1 \mathrm{~mm}$ margin to $>2 \mathrm{~mm}$ margin, and the most common PTV margin used in our series was $2 \mathrm{~mm}$. In the trial conducted at MD Anderson, the PTV margin was $1 \mathrm{~mm} .{ }^{22}$ It is possible that this difference in margin may affect LC, although there remains no randomized evidence available describing the ideal PTV margin that would optimize LC and minimize toxicity.

Although the MD Anderson and N107C.CEC. 3 studies stratified preresection tumors by $<3$ or $\geq 3 \mathrm{~cm}$, the inclusion of large resection cavities were altogether limited given their exclusion criteria of resection cavities $>4 \mathrm{~cm}$ and $5 \mathrm{~cm}$, respectively. ${ }^{15,22}$ Accordingly, many of the patients in our $>4 \mathrm{~cm}$ cohort may have resection cavities too large for enrollment for these trials, given previous authors' concerns with decreased LC rates in these resection cavities. Our series suggests that even these larger resection cavities have promising $\mathrm{LC}$ rates 
when adequately dosed. Consequently, we believe that these larger resection cavities may be safely enrolled in future clinical trials if appropriate dosing considerations are made.

This study has several limitations generally associated with retrospective studies. There may exist selection bias for more favorable patients with large resection cavities to be treated at our institution; however, the baseline characteristics in our series do not demonstrate measurable differences in the wide array of patient and disease characteristics known to affect treatment outcomes. Additionally, because of the rarity of SRS treatment to large tumor resection cavities, only 27 patients in our series had preresection cavities $>4 \mathrm{~cm}$. Despite our small patient cohort size, this is the only report to our knowledge that compares LC outcomes in tumors $\geq 4 \mathrm{~cm}$. Strengths of this study include homogenous patient treatment methods and the use of cumulative incidence with death as a competing risk to more accurately estimate event rates in this setting where death without the event can be common. Our study reports 3-dimensional volumetric data over 2-dimensional axial measurements because volumetric measurements more accurately represents treatment volume and resection cavities generally lose their spherical shape following resection. ${ }^{24} \mathrm{We}$ published the preoperative tumor measurements so that the findings of this study can be used in multidisciplinary decision-making at which time the tumor has not undergone resection. We have also published the postoperative PTVs as this information more directly corresponds to rates of LC and toxicity. ${ }^{25}$ We believe the preoperative and postoperative tumor characteristics are complementary and both should be considered in treatment planning.

In summary, our series suggests that postoperative SRS alone to resection cavities from brain metastases $>4 \mathrm{~cm}$ is a viable strategy with promising LC rates and acceptable toxicity. We believe that these data may be useful in treatment decision making for these patients as an alternative to whole brain irradiation. Additionally, patients with resection cavities from large metastases may be safely included in future prospective trials, particularly in those using hypofractionated SRS.

\section{Acknowledgments}

Sources of support: Research reported in this publication was supported in part by the Biostatistics and Bioinformatics Shared Resource of Winship Cancer Institute of Emory University and the National Institutes of Health/National Cancer Institute under award number P30CA138292.

The content is solely the responsibility of the authors and does not necessarily represent the official views of the National Institutes of Health.

\section{References}

1. Nayak L, Lee EQ, Wen PY. Epidemiology of brain metastases. Curr Oncol Rep. 2012; 14:48-54. [PubMed: 22012633]

2. Patel KR, Lawson DH, Kudchadkar RR, et al. Two heads better than one? Ipilimumab immunotherapy and radiation therapy for melanoma brain metastases. Neuro-Oncology. 2015; 17:1312-1321. [PubMed: 26014049]

3. Kocher M, Soffietti R, Abacioglu U, et al. Adjuvant whole-brain radiotherapy versus observation after radiosurgery or surgical resection of one to three cerebral metastases: Results of the EORTC 22952-26001 study. J Clin Oncol. 2011; 29:134-141. [PubMed: 21041710] 
4. Patchell RA, Tibbs PA, Regine WF, et al. Postoperative radiotherapy in the treatment of single metastases to the brain: A randomized trial. JAMA. 1998; 280:1485-1489. [PubMed: 9809728]

5. Patchell RA, Tibbs PA, Walsh JW, et al. A randomized trial of surgery in the treatment of single metastases to the brain. N Engl J Med. 1990; 322:494-500. [PubMed: 2405271]

6. Patel KR, Prabhu RS, Kandula S, et al. Intracranial control and radiographic changes with adjuvant radiation therapy for resected brain metastases: Whole brain radiotherapy versus stereotactic radiosurgery alone. J Neuro-Oncol. 2014; 120:657-663.

7. Brown PD, Jaeckle K, Ballman KV, et al. Effect of radiosurgery alone vs radiosurgery with whole brain radiation therapy on cognitive function in patients with 1 to 3 brain metastases: A randomized clinical trial. JAMA. 2016; 316:401-409. [PubMed: 27458945]

8. Chang EL, Wefel JS, Hess KR, et al. Neurocognition in patients with brain metastases treated with radiosurgery or radiosurgery plus whole-brain irradiation: A randomised controlled trial. Lancet Oncol. 2009; 10:1037-1044. [PubMed: 19801201]

9. Patel, KR., Burri, SH., Boselli, D., et al. Comparing pre-operative stereotactic radiosurgery (srs) to post-operative whole brain radiation therapy (WBRT) for resectable brain metastases: A multiinstitutional analysis. J Neuro-Oncol. May 10, 2017 http://dx.doi.org/10.1007/s11060-016-2334-3, [e-pub ahead of print]

10. Andrews DW, Scott CB, Sperduto PW, et al. Whole brain radiation therapy with or without stereotactic radiosurgery boost for patients with one to three brain metastases: Phase III results of the RTOG 9508 randomised trial. Lancet. 2004; 363:1665-1672. [PubMed: 15158627]

11. Aoyama H, Shirato H, Tago M, et al. Stereotactic radiosurgery plus whole-brain radiation therapy vs stereotactic radiosurgery alone for treatment of brain metastases: A randomized controlled trial. JAMA. 2006; 295:2483-2491. [PubMed: 16757720]

12. Brennan C, Yang TJ, Hilden P, et al. A phase 2 trial of stereotactic radiosurgery boost after surgical resection for brain metastases. Int J Radiat Oncol Biol Phys. 2014; 88:130-136. [PubMed: 24331659]

13. Mathieu D, Kondziolka D, Flickinger JC, et al. Tumor bed radiosurgery after resection of cerebral metastases. Neurosurgery. 2008; 62:817-823. [PubMed: 18414136]

14. Soltys SG, Adler JR, Lipani JD, et al. Stereotactic radiosurgery of the postoperative resection cavity for brain metastases. Int J Radiat Oncol Biol Phys. 2008; 70:187-193. [PubMed: 17881139]

15. Brown PD, Ballman KV, Cerhan J, et al. N107c/cec.3: A phase III trial of post-operative stereotactic radiosurgery (SRS) compared with whole brain radiotherapy (WBRT) for resected metastatic brain disease. Int J Radiat Oncol Biol Phys. 2016; 96:937.

16. Kumar AJ, Leeds NE, Fuller GN, et al. Malignant gliomas: MR imaging spectrum of radiation therapy- and chemotherapy-induced necrosis of the brain after treatment. Radiology. 2000; 217:377-384. [PubMed: 11058631]

17. Kalbfleisch, JDP., Prentice, RL. The Statistical Analysis of Failure Time Data. Hoboken, NJ: Wiley-Interscience; 2002.

18. Patel KR, Burri SH, Asher AL, et al. Comparing preoperative with postoperative stereotactic radiosurgery for resectable brain metastases: A multi-institutional analysis. Neurosurgery. 2016; 79:279-285. [PubMed: 26528673]

19. Mahajan A, Ahmed S, Li J, et al. Postoperative stereotactic radiosurgery versus observation for completely resected brain metastases: Results of a prospective randomized study. Comparing preoperative with postoperative stereotactic radiosurgery for resectable brain metastases: A multiinstitutional analysis. 2016; 96:S2.

20. Prabhu R, Shu HK, Hadjipaayis C, et al. Current dosing paradigm for stereotactic radiosurgery alone after surgical resection of brain metastases needs to be optimized for improved local control. Int J Radiat Oncol Biol Phys. 2012; 83:e61-e66. [PubMed: 22516387]

21. Eaton BR, LaRiviere MJ, Kim S, et al. Hypofractionated radiosurgery has a better safety profile than single fraction radiosurgery for large resected brain metastases. J Neuro-Oncol. 2015; 123:103-111.

22. Minniti G, Clarke E, Lanzetta G, et al. Stereotactic radiosurgery for brain metastases: Analysis of outcome and risk of brain radionecrosis. Radiat Oncol. 2011; 6:48. [PubMed: 21575163] 
23. Atalar B, Modlin LA, Choi CY, et al. Risk of leptomeningeal disease in patients treated with stereotactic radiosurgery targeting the postoperative resection cavity for brain metastases. Int $\mathbf{J}$ Radiat Oncol Biol Phys. 2013; 87:713-718. [PubMed: 24054875]

24. Shah JK, Potts MB, Sneed PK, Aghi MK, McDermott MW. Surgical cavity constriction and local progression between resection and adjuvant radiosurgery for brain metastases. Cureus. 2016; 8:e575. [PubMed: 27226936]

25. Shaw E, Scott C, Souhami L, et al. Single dose radiosurgical treatment of recurrent previously irradiated primary brain tumors and brain metastases: Final report of RTOG protocol 90-05. Int J Radiat Oncol Biol Phys. 2000; 47:291-298. [PubMed: 10802351] 


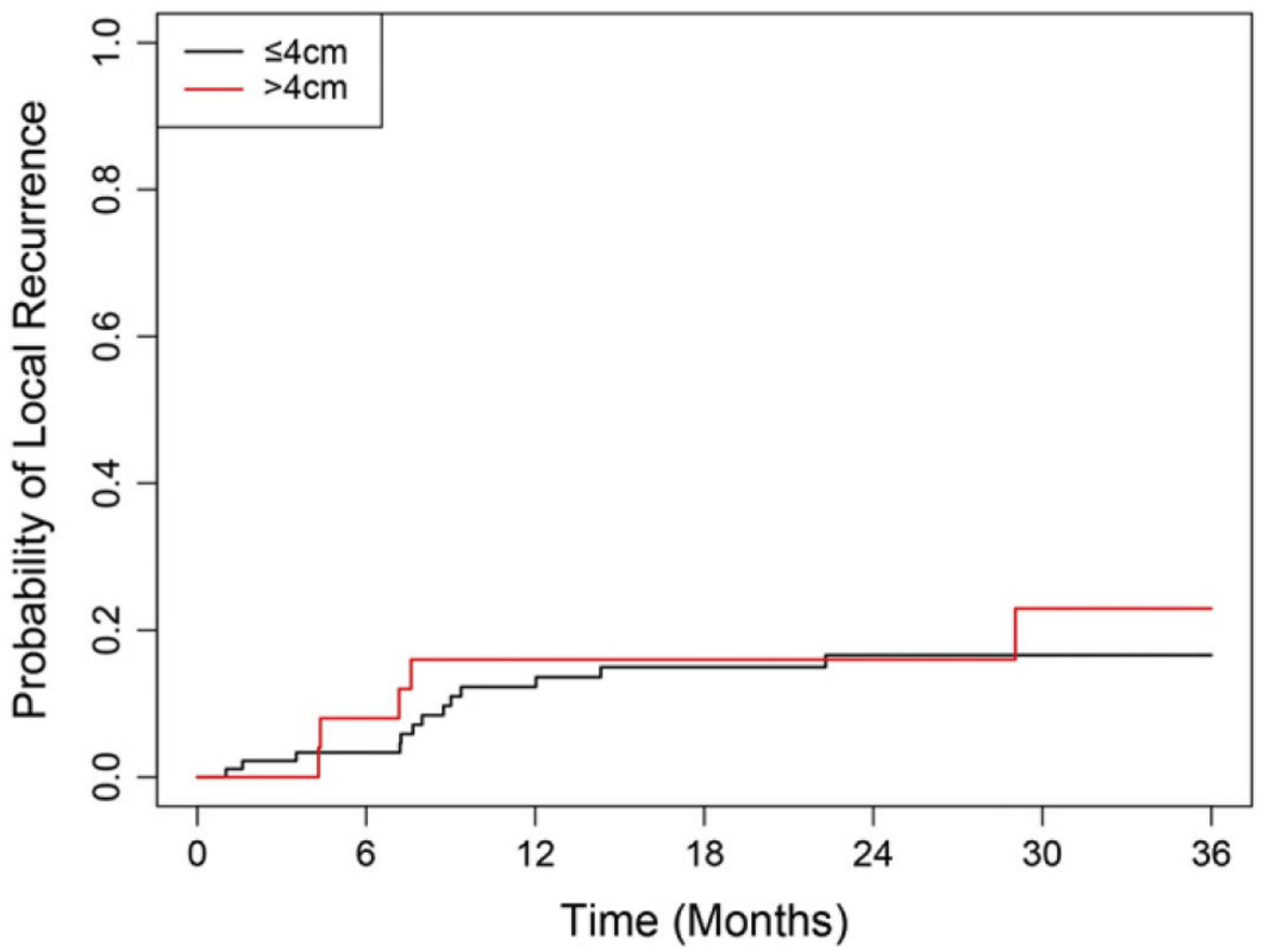

Number at Risk

Figure 1.

Cumulative incidence model for local recurrence for lesions $\leq 4 \mathrm{~cm}$ and lesions $>4 \mathrm{~cm}$. 


\section{Table 1}

Baseline patient and tumor characteristics

\begin{tabular}{|c|c|c|c|}
\hline & $\begin{array}{l}\text { Tumors } \leq 4 \mathrm{~cm} \\
\mathrm{n}=90(77 \%)\end{array}$ & $\begin{array}{l}\text { Tumors }>4 \mathrm{~cm} \\
\mathrm{n}=27(23 \%)\end{array}$ & $P$ value \\
\hline Maximum dimension $(\mathrm{cm})$ & Median, 2.8 (95\% CI, 2.6-2.9) & Median 4.7 (95\% CI: 4.6-5.2) & $<.001$ \\
\hline Age (minimum-maximum) & $58(20-77)$ & $56(32-83)$ & .54 \\
\hline \multirow[t]{2}{*}{ Sex } & Men: $38(42 \%)$ & Men: $10(37 \%)$ & .63 \\
\hline & Women: $52(58 \%)$ & Women: 17 (63\%) & \\
\hline \multirow[t]{6}{*}{ Primary site histology } & NSCLC: $41(46 \%)$ & NSCLC: $11(41 \%)$ & .9 \\
\hline & Breast: $17(19 \%)$ & Breast: $5(19 \%)$ & \\
\hline & Melanoma: $18(20 \%)$ & Melanoma: $6(22 \%)$ & \\
\hline & RCC: $5(6 \%)$ & RCC: $3(11 \%)$ & \\
\hline & GI: $1(1 \%)$ & GI: $0(0 \%)$ & \\
\hline & Other: $8(9 \%)$ & Other: $2(7 \%)$ & \\
\hline \multirow[t]{3}{*}{ Recursive partitioning analysis class } & I: $35(39 \%)$ & I: $12(44 \%)$ & .61 \\
\hline & II: $52(58 \%)$ & II: 13 (48\%) & \\
\hline & III: $3(3 \%)$ & III: $2(7 \%)$ & \\
\hline \multirow{7}{*}{$\begin{array}{l}\text { Tumor specific-graded prognostic assessment } \\
\text { Score }\end{array}$} & 1.0: $2(2 \%)$ & 1: $0(0 \%)$ & .11 \\
\hline & $1.5: 12(13 \%)$ & $1.5: 6(22 \%)$ & \\
\hline & $2: 8(9 \%)$ & $2: 4(15 \%)$ & \\
\hline & $2.5: 35(39 \%)$ & $2.5: 3(11 \%)$ & \\
\hline & 3: $19(21 \%)$ & 3: $7(26 \%)$ & \\
\hline & $3.5: 11(12 \%)$ & $3.5: 6(22 \%)$ & \\
\hline & 4: $3(3 \%)$ & $4: 1(4 \%)$ & \\
\hline \multirow[t]{2}{*}{ Framed vs frameless } & Framed: $59(66 \%)$ & Framed: $14(52 \%)$ & .2 \\
\hline & Frameless: $31(34 \%)$ & Frameless: $13(48 \%)$ & \\
\hline \multirow[t]{2}{*}{ Prescribed total dose (Gy) } & Median: 18 (95\% CI, 19-23) & Median: 20 (95\% CI, 19-24) & .85 \\
\hline & Mean: 21.1 & Mean: 21.5 & \\
\hline \multirow[t]{5}{*}{ Prescribed $\mathrm{BED}_{10}(\mathrm{~Gy})$} & Mean: 46.8 (95\% CI, 45.2-48.4) & Mean: 42.5 (95\% CI, 39.7-45.4) & .007 \\
\hline & 1 fraction: 46.8 (95\% CI, 44.8-48.8) & 1 fraction: 40.9 (95\% CI, 36.7-45.2) & \\
\hline & 3 fractions: 37.6 (95\% CI, 33.1-42.1) & 3 fractions: 35.7 (95\% CI, 29.3-42.1) & \\
\hline & 4 fractions: 38.4 (95\% CI, NA) & 4 fractions: $38.4(95 \% \mathrm{CI}, \mathrm{NA})$ & \\
\hline & 5 fractions: $49.2(95 \%$ CI, 47.3-51.1) & 5 fractions: 46.5 (95\% CI, 43.8-49.2) & \\
\hline \multirow[t]{4}{*}{ Number of fractions } & Single: $66(73 \%)$ & Single: $14(52 \%)$ & .21 \\
\hline & $3: 4(4 \%)$ & $3: 2(7 \%)$ & \\
\hline & $4: 1(1 \%)$ & $4: 1(4 \%)$ & \\
\hline & 5: $19(21 \%)$ & 5: $10(37 \%)$ & \\
\hline \multirow[t]{2}{*}{ Extent of surgical resection } & STR: $22(24 \%)$ & STR: $14(51 \%)$ & $<.01$ \\
\hline & GTR: $68(76 \%)$ & GTR: $13(48 \%)$ & \\
\hline \multicolumn{4}{|l|}{ PTV margin (mm) } \\
\hline \multirow[t]{3}{*}{ Frameless } & $<2 \mathrm{~mm}: 12(39 \%)$ & $<2 \mathrm{~mm}: 3(23 \%)$ & .15 \\
\hline & 2-3 mm: $18(58 \%)$ & $2-3$ mm: 9 (69\%) & \\
\hline & $>3 \mathrm{~mm}: 1(3 \%)$ & $>3$ mm: $1(8 \%)$ & \\
\hline
\end{tabular}




\begin{tabular}{|c|c|c|c|}
\hline & $\begin{array}{l}\text { Tumors } \leq 4 \mathrm{~cm} \\
\mathrm{n}=90(77 \%)\end{array}$ & $\begin{array}{l}\text { Tumors }>4 \mathrm{~cm} \\
\mathrm{n}=27(23 \%)\end{array}$ & $P$ value \\
\hline \multirow[t]{3}{*}{ Framed } & $<2 \mathrm{~mm}: 29(49 \%)$ & $<2 \mathrm{~mm}: 9(64 \%)$ & .14 \\
\hline & $2-3 \mathrm{~mm}: 30(51 \%)$ & $2-3 \mathrm{~mm}: 5(36 \%)$ & \\
\hline & $>3 \mathrm{~mm}: 0(0 \%)$ & $>3 \mathrm{~mm}: 0(0 \%)$ & \\
\hline $\operatorname{GTV}\left(\mathrm{cm}^{3}\right)$ & Mean: 11.1 (95\% CI, 9.5-12.7) & Mean: 20.8 (95\% CI, 16.4-25.1) & $<.001$ \\
\hline PTV treated $\left(\mathrm{cm}^{3}\right)$ & Mean: $18.6(95 \%$ CI, 16.0-21.2) & Mean: 33.7 (95\% CI, 26.6-40.1) & $<.001$ \\
\hline
\end{tabular}

BED10, biologically effective dose at alpha/beta ratio of 10; CI, confidence interval; GI, gastrointestinal; GTR, gross total resection; GTV, gross tumor volume; NA, not available; NSCLC, non-small cell lung cancer; PTV, planning tumor volume; RCC, renal cell cancer; STR, subtotal resection. 


\section{을 \\ }

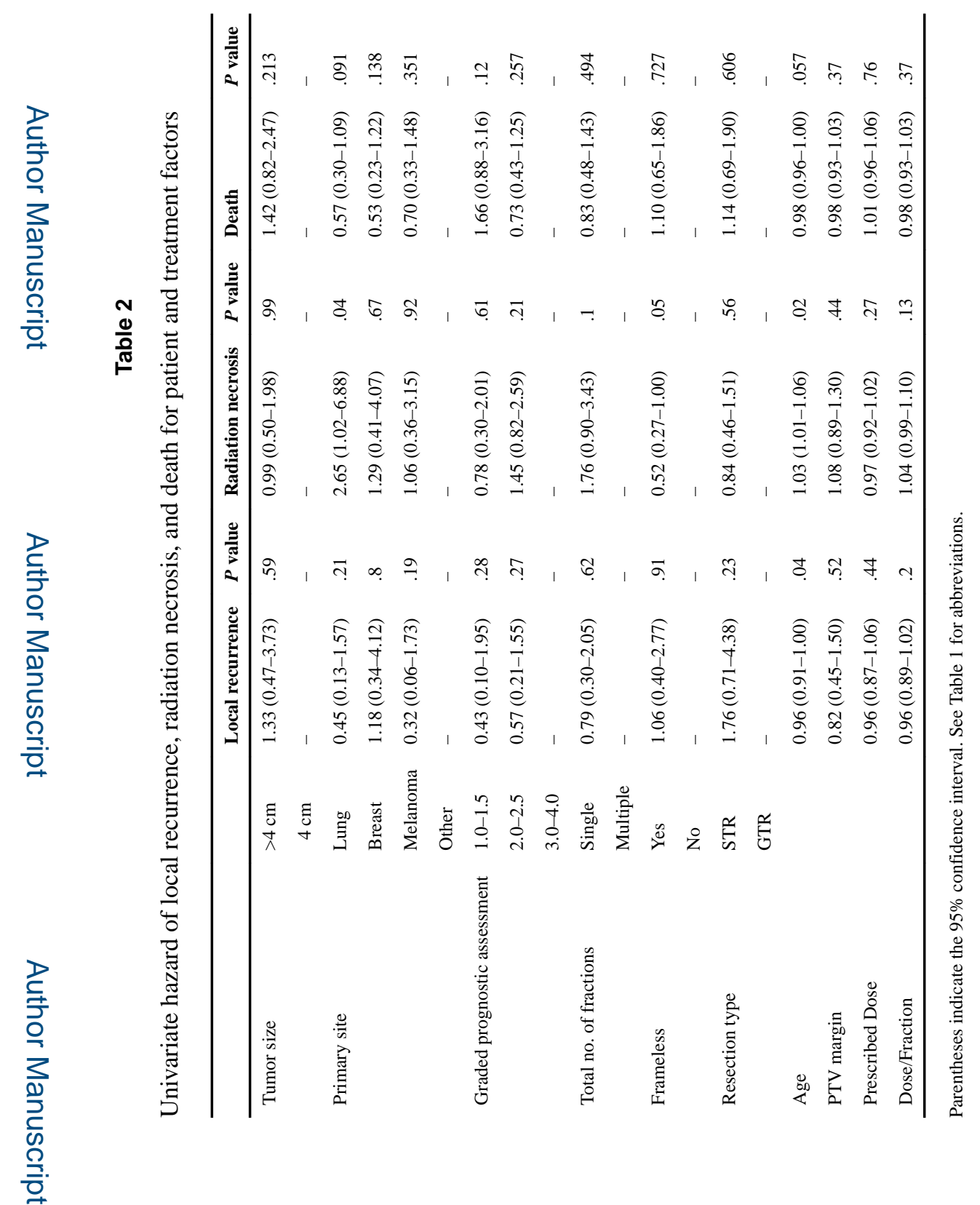

Pract Radiat Oncol. Author manuscript; available in PMC 2018 May 12. 